\title{
Outcomes in patients with chronic uveitis: which factors matter to patients? A qualitative study
}

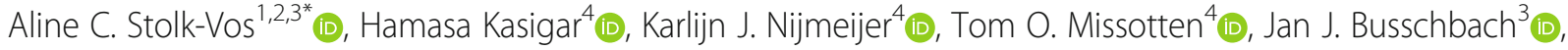 \\ Joris J. van de Klundert ${ }^{2,5}$ (D) and Leonieke W. Kranenburg ${ }^{3}$ (D)
}

\begin{abstract}
Purpose: Outcome measurements currently used in chronic uveitis care fail to cover the full patient perspective. The aim of this study is to develop a conceptual model of the factors that adult patients with chronic uveitis consider to be important when evaluating the impact of their disease and treatment.

Methods: A qualitative study design was used. Twenty chronic uveitis patients were recruited to participate in two focus groups. Data were transcribed verbatim and analysed using thematic analysis in ATLAS.ti.

Results: Coding of the transcripts resulted in a total of 19 codes divided over five themes: 1 ) disease symptoms and treatment; 2) diagnosis and treatment process; 3) impact on daily functioning; 4) emotional impact; and 5) treatment success factors.
\end{abstract}

Conclusion: The conceptual model resulting from this study can contribute to the development of future uveitis specific measures in adults.

Keywords: Patient reported outcome measures (MeSH), Surveys and questionnaires (MeSH), Ophthalmology (MeSH), Quality of health care (MeSH), Qualitative research (MeSH), Quality of life (MeSH), Uveitis (MeSH)

\section{Introduction}

Chronic uveitis, a disease characterized by intraocular inflammations, is a complex and variable eye condition potentially leading to blindness and affecting adults in the working age group [1]. It is often treated systemically. Patients diagnosed with chronic uveitis not only have problems with the chronicity of the disease and side effects of the medication, but also with the unpredictability of inflammations, transient visual acuity, inflammatory activity changes, and sometimes unexpected

\footnotetext{
* Correspondence: a.stolk@eyehospital.nl

${ }^{1}$ Rotterdam Ophthalmic Institute, Schiedamse Vest 160, Rotterdam 3011 BH, The Netherlands

${ }^{2}$ Erasmus School of Health Policy \& Management, Erasmus University Rotterdam, Rotterdam, The Netherlands

Full list of author information is available at the end of the article
}

complications of the disease and the medication used [2-4].

A previous review found high heterogeneity of outcome measures that are currently used for the evaluation of uveitis treatment. Common outcome measures were classified in several domains: 1) disease activities, 2) visual function, and 3) tissue damage or other disease complications. However, those clinical outcomes are limited in the extent to which they inform us on how patients experience the impact of their disease. For example, patients' evaluation of their ability to conduct daily activities, such as reading and driving, are not included $[5,6]$. As chronic uveitis can have a huge impact on healthrelated quality of life [2-4], currently used primary outcome measures may therefore fall short of appropriately addressing what patients consider as most important [7]. 
Commonly used instruments for patients with chronic uveitis are the SF-36 Health Survey [8] to measure health-related quality of life in a generic way and the 25item National Eye Institute Visual Function Questionnaire (NEI-VFQ-25) [6] to measure quality of life in a domain specific way, i.e. vision-related quality of life. However, as these instruments are not specifically developed for the complex and variable condition chronic uveitis $[9,10]$, the resulting assessment may be incomplete. There is a disease specific instrument developed for uveitis, EYE-Q [11], but this instrument is meant for a paediatric population, while chronic uveitis is most prevalent in adults.

The development of an instrument for the adult population firstly requires understanding which factors chronic uveitis patients consider relevant. So far, there has been published no substantial qualitative in-depth research effort that focused on the patient perspectives on disease and treatment [7]. The aim of the current study is to develop a conceptual model of the factors that adult patients with chronic uveitis consider to be important when evaluating the impact of their disease and treatment. This conceptual model can contribute to the development of future uveitis specific measures in adults.

\section{Methods}

\section{Study design}

To determine the factors that patients with chronic uveitis consider important when evaluating the impact of their disease and treatment, we used a qualitative study design based on focus group discussion [12]. Such a focus group approach is recommended in several relevant guidelines like those of ISPOR [13] and the FDA [14], in order to assure that all factors of disease and treatment that patients consider important are determined.

This study is part of TopZorg, a project subsidized by the Dutch Organisation for Health Research and Development (ZonMw). TopZorg aims to stimulate scientific research on highly specialized care in non-academic hospitals. This study has been approved by the medical ethics committee METC of Erasmus Medical Center (MEC2017-557).

\section{Study sample}

We invited chronic uveitis patients of The Rotterdam Eye Hospital to participate in this study. To include a representative cross section of all chronic uveitis patients, patients were selected from the registries by means of stratified random sampling. Strata used were type of chronic uveitis, time since diagnose, gender and age. The inclusion criteria were 1) diagnosed with chronic uveitis [15] for more than 3 months; 2) having anterior segment uveitis, posterior segment uveitis, or panuveitis. We used the Dutch reimbursement codes 502 and 503, respectively referring to anterior segment uveitis and to posterior segment uveitis (intermediate and posterior) and panuveitis. These codes match with ICD-10 codes H20.x, H30.x and H44.1; 3) 18 years or older. We excluded patients who did not have a good command of the Dutch language. Two focus groups, one with 9 and one with 11 participants, were conducted to draw out different perspectives and generate discussion, thereby allowing each person to talk in detail about their perspective [16]. Selected patients received a letter with study information signed by their treating ophthalmologist. They were subsequently contacted by phone and invited to participate in the focus groups. Besides the selected patients, we invited the chairman of the uveitis patient association from the Dutch Eye Patient Association. The chairman met the inclusion criteria. All participants signed informed consent.

\section{Data collection}

Focus group data were collected between February 2018 and March 2018. The focus groups took place at The Rotterdam Eye Hospital and were chaired by a moderator (HK). This moderator facilitated open exchange among participants. The moderator made use of a predefined semi-structured topic list with open-ended questions (Additional file 1) to structure the discussion and to prevent missing relevant topics. The topic list was based on a literature review and on input from representatives of the Dutch uveitis patient association. An observer (LK) was present to observe non-verbal communication and support the moderator if necessary. At the start of discussion, participants were asked to be respectful to each other, and the moderator emphasized the importance of hearing from every participant. The focus groups had a duration of $2 \mathrm{~h}$, including a $15 \mathrm{~min}$ break. Focus groups were audioand video recorded and transcribed verbatim.

\section{Data analysis}

Thematic analysis was conducted applying a deductive approach to theme generation. Themes were selected based on the questions in the topic list (Additional file 1). Two researchers (LK and AS) carefully read the transcripts. Each of the two independently developed a structured analysis framework consisting of preliminary themes and codes. They compared their frameworks to reach consensus. Thereafter, two researchers (HK and AS) independently indexed the transcripts line by line according to this framework using ATLAS.ti [17]. Coders used memos for comments during coding. When coding was finished and the code 'other' was used, this code was renamed into a new or existing codename best reflecting the contents of the otherwise uncategorised transcripts. Coders compared their coding and discussed 
until consensus was achieved [18-20]. Subsequently, the framework was refined by removing, adding or combining codes in order to maximise internal homogeneity and external heterogeneity [21]. The final framework is added in Additional file 2. After coding was finished, the cohesion and inter-relations between codes were analysed and visually depicted in a map.

\section{Additional external validation}

After conducting two focus groups we concluded that data saturation was achieved, i.e. no new information emerged in the second group. As there was discussion within the research group whether two focus groups might look insufficient to achieve data saturation, we decided to conduct an additional external validity check by asking chronic uveitis patients to reflex on the results, and test whether they consider the results to be complete. Such a validity check is a recommended method by Green \& Thorogood [22]. More specifically, we presented the findings to six members of the uveitis patient division of the Dutch Eye Patient Association, asking them whether they concurred with the topics in the structured analysis matrix (Additional file 2), which of these topics they considered to be important, and to note missing topics.

\section{Results}

\section{Participants}

There were two focus group sessions involving 20 participants in total. The characteristics of the participants are described in Table 1.

\section{Structure}

Thematic analysis of the focus groups yielded five central themes characterising factors that patients with chronic uveitis consider to be important when evaluating the impact of their disease: 1) disease symptoms and characteristics; 2) diagnosis and treatment process; 3) impact on daily functioning; 4) emotional impact; and 5) treatment success factors. Table 2 lists those themes and underlying codes including a summary of the content.

\section{Theme 1 disease symptoms and treatment}

The symptoms experienced and various treatment options were discussed at length. Patients reported symptoms related to vision and symptoms related to pain and discomfort. The extent to which they experienced symptoms depended on their personal condition and differed strongly between patients, e.g. from no vision to very good vision and from no pain at all to unbearable pain.

Further, patients experienced difficulties attributing symptoms to chronic uveitis, since most patients suffered from comorbid conditions (comorbidity). As symptoms and comorbidity were different among patients, medication use and side effects of that medication use also differed between patients. Treatments given to patients included steroids, immunotherapy and biologicals. Medication use received much attention in the discussions. Patients were especially interested in each other's experiences with various types of medication, ways of taking medication - infuse, tablet, injection, drops - and dosage. Besides medication use, patients also mentioned surgeries and hospitalizations, however they did so only in relation to comorbidity and not to uveitis.

\section{Theme 2 diagnosis and treatment process}

Most patients commented that it took long until they were correctly diagnosed with uveitis. This diagnostic process was characterized by slow referrals from the general practitioner to specialist care, many examinations of which many were unnecessary -, and even misdiagnosis. For instance, a patient said: "Actually, my optician discovered it by chance. He said: there is an inflammation in your eye. Then it took me a long time to finally get my primary care doctor's permission. And, indeed, examination has shown that it was sarcoidosis".

Even when patients were diagnosed with uveitis, they experienced a poor recognition of uveitis by the general practitioner, emergency care physicians, and ophthalmology residents in cases where their own specialist was not available. This poor recognition resulted in inadequate examinations and medication prescriptions or in long time to treatment, as is illustrated by the following

Table 1 Patients' characteristics participants' focus group

\begin{tabular}{llll}
\hline & Focus group 1 & Focus group 2 & Total \\
\hline $\mathrm{N}$ & 11 & 9 & 20 \\
Women, $\mathrm{n}(\%)$ & $7(64)$ & $5(56)$ & $12(60)$ \\
Age in years, mean (range) & $56(32-74)$ & $53(38-65)$ & $55(32-74)$ \\
Diagnose code, n (\%) & & $5(56)$ & $10(50)$ \\
- ICD-10 H20.x Anterior segment & $5(45)$ & $2(22)$ & $6(30)$ \\
- ICD-10 H30.x Posterior segment & $4(36)$ & $2(22)$ & $4(20)$ \\
- ICD-10 H44.1 Panuveitis & $2(18)$ & $7(1-14)$ & $9(1-14)$ \\
Years since diagnosis, median (range) & $10(3-13)$ & & \\
\hline
\end{tabular}



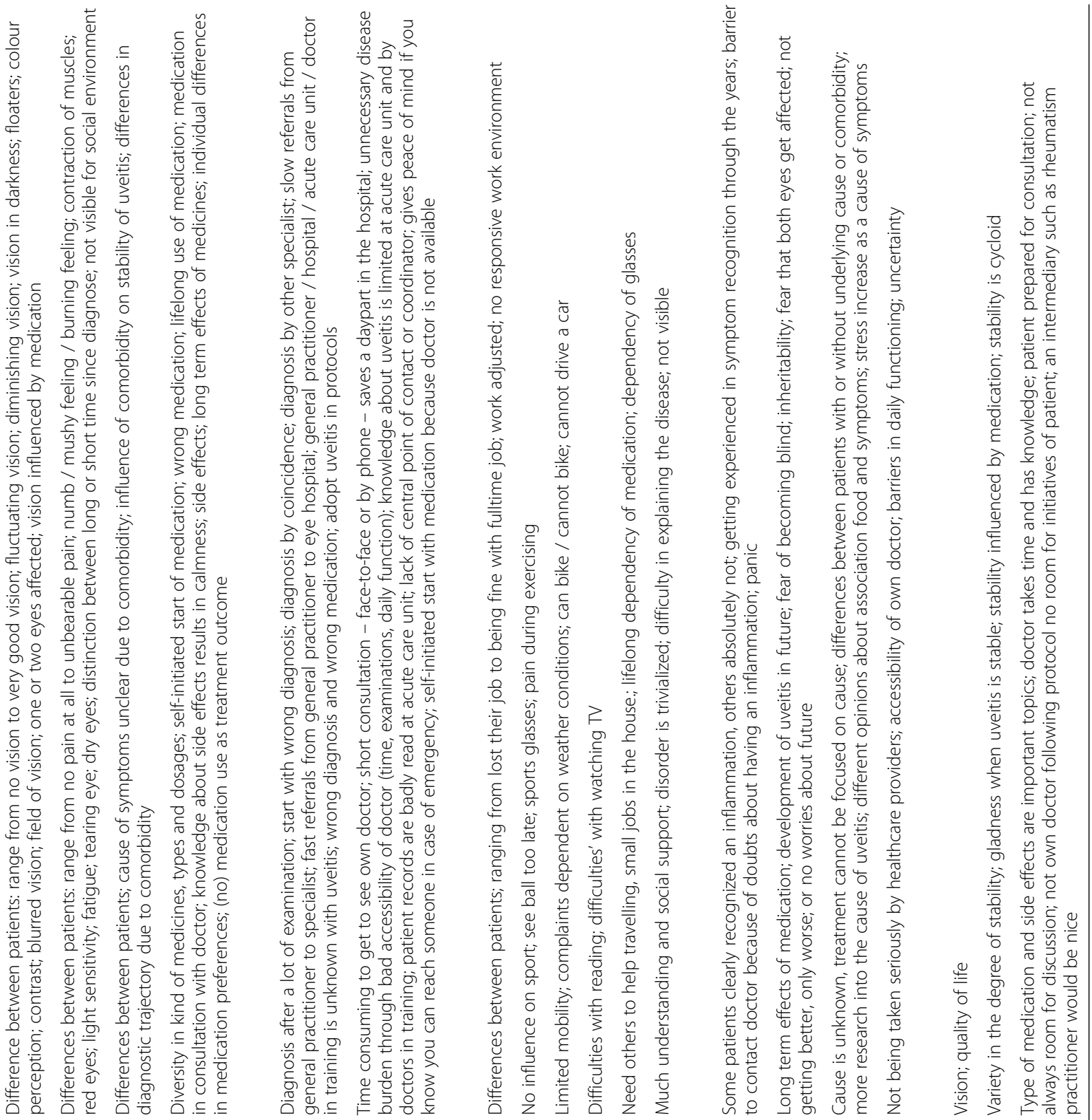
quote: "And then you get there at the emergency department. And then you get all kinds of examinations with which you are even worse off. Sometimes also with medication that are of no use. When I get to my own ophthalmologist, I have the correct diagnosis and the right medication within five minutes, and I am done within five minutes".

Further, patients reported that they experienced difficulties in reaching their own uveitis specialist. They experienced the limited accessibility as an unnecessary disease burden. "That you are in direct contact with him [own uveitis specialist], [...] you just want to be able to act quickly and now you are actually stopped by how it is organized.".

\section{Theme 3 impact on daily functioning}

Patients varied strongly in the impact chronic uveitis had on their daily function, including activities such as employment, sports, mobility, and watching TV or reading. For example, one patient reported to have lost her job because of chronic uveitis, by contrast, another patient reported to do fine with her fulltime job. Further, patients discussed different patterns of dependency including dependency on other people, lifelong dependency on medication, and dependency on devices. To illustrate, one patient said: "Yes, even if you just arrived in southern France and you have to say [to your spouse] the next morning: [we have to] go back again, because I have to go to Rotterdam. That has happened to me often".

Further, the impact on daily functioning depends on support patients experience within relationships. Some patients experienced much understanding from their social environment, while others felt that their environment downplayed the severity of their disease which enlarged the impact of disease burden.

\section{Theme 4 emotional impact}

Patients highlight several emotional consequences of chronic uveitis. A main topic is the uncertainty patients experienced because of the unpredictability of the disease. We distinguished three different kinds of uncertainty. The first is uncertainty about the inflammation. Some patients could clearly recognize an inflammation, while others were unable to do so. Patients who experience difficulties in recognition made remarks like: "But in this case: do I have it or not? And then you cross that threshold to go to a doctor. That for me is the uncertainty." Secondly, there is uncertainty about the future: the long-term effects of medication, the development of chronic uveitis, the fear of becoming blind and questions regarding inheritability. For instance, a patient said: "That is really the rottenest thing I have, I think. Most frightening $[. .$.$] and uh, yes, I am afraid that my other$ eye, my good eye, will be like that too." Lastly, patients perceive uncertainty about causes of complaints. It involves doubt about whether it is the uveitis that causes certain complaints or whether those results from a comorbid disorder.

In addition, patients often named stress as an important factor. The emotional stress may be caused by the feeling of not being taken seriously by health professionals, by lack of timely access to their own ophthalmologist, by experienced barriers in daily functioning, or by the dependency caused by the chronic uveitis.

\section{Theme 5 treatment success factors}

Treatment success factors emerged as a fifth theme. Patients perceived three main treatment success factors: 1) outcome - in terms of improvement in vision and/or quality of life; 2) stability - in terms of happiness when the uveitis is under control; and 3) the degree of shared decision making between patient and ophthalmologist - in terms of having enough time for consultation, sharing knowledge and experiences, and being able to exert influence on decision making on medication use. To illustrate stability, one patient mentioned: "eh I also see my treatment as very successful. It has taken eight nine years, continuous bleeding, flares and inflammations in my eye. Nerves and it all. That has now completely calmed down. No bleeding, no inflammation. So, I am a happy person." Medication use and side effects were important topics in shared decision making. Patients noticed that shared decision making was not always there, whereas they would have liked otherwise to experience their treatment as successful.

\section{Cohesion between themes and codes}

The cohesion and inter-relations between themes and codes is depicted in Fig. 1. Medication and side effects is placed in the middle indicating its central role. It is closely related to accessibility and shared decision making. This is because (questions about) medication use are an important reason for the desire for easily accessible care and an important topic during consultations according to patients. Further, it is notable that codes belonging to one and the same theme are clustered close together, which indicates the uniformity of defined themes (see Fig. 1). Lastly, we noticed that the code stress came up in between codes across various themes underwriting the importance of stress due to chronic uveitis in patients' daily life.

\section{Additional external validation}

Six members of the uveitis patient division of the Dutch Eye Patient Association took part in the additional external validity check to maximize validity (Table 3 ). Results were in line with our findings and no new topics came up. 


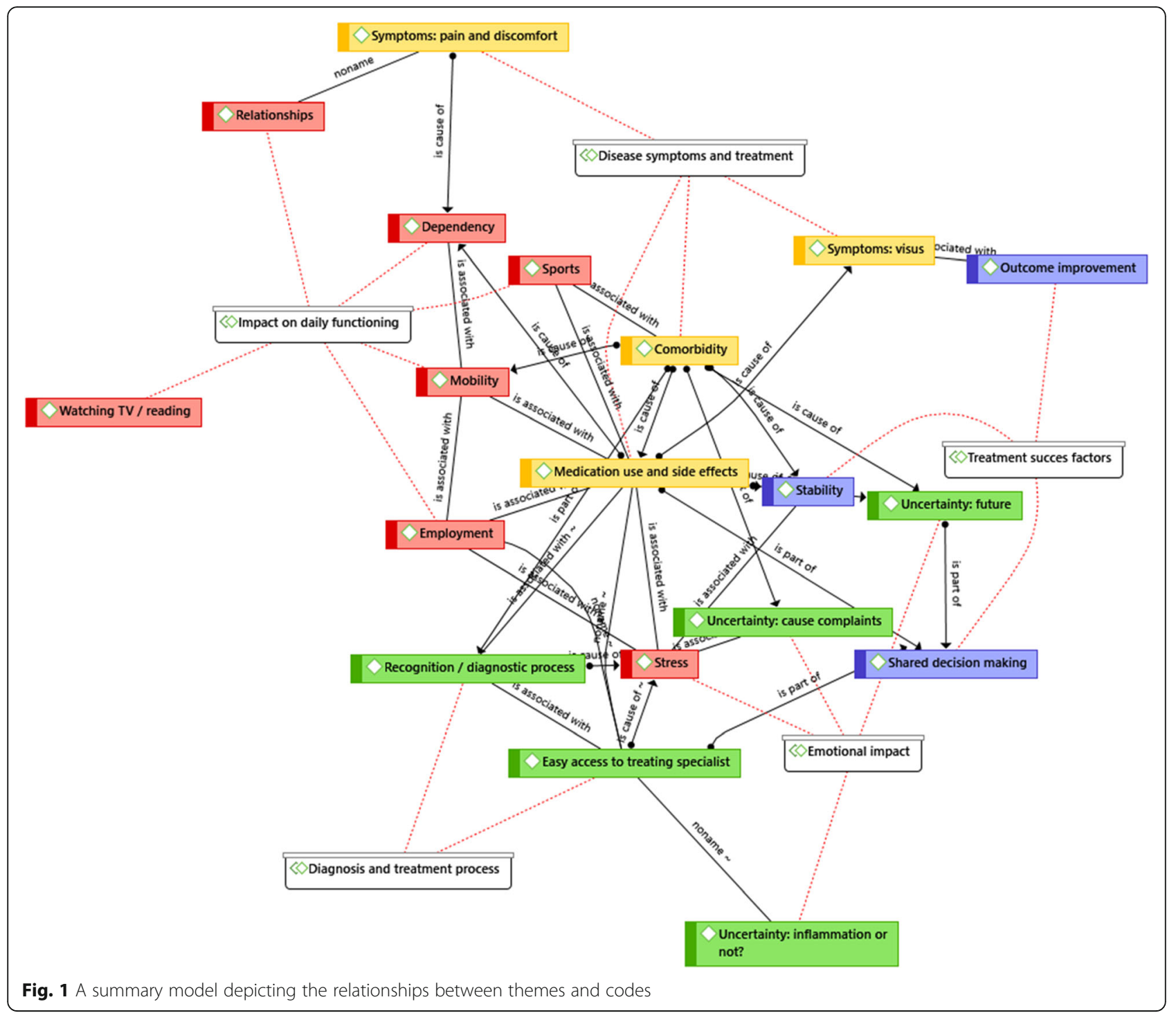

\section{Discussion}

This study shows a conceptual model with five themes that patients with chronic uveitis consider to be of importance when evaluating the impact of their disease and treatment: disease symptoms and treatment, diagnosis and treatment process, impact on daily functioning, emotional impact, and treatment success factors.

Table 3 Patients' characteristics of members from uveitis patient association involved in validity check

\begin{tabular}{ll}
\hline & Total \\
\hline Women, $\mathrm{n}(\%)$ & 6 \\
Age in years, mean (range) & $4(67)$ \\
Years since diagnosis, median (range) & $55(43-67)$ \\
\hline
\end{tabular}

Therefore, we recommend these five themes to be included in the development of future uveitis specific measures in adults.

Considering how these themes relate to the most frequently used instruments, SF-36 and VFQ-25, we notice that they only partly cover the patient perspective. The generic SF-36 may measure the theme 'impact on daily function' accurate yet fails to cover uveitis-specific outcomes in the themes 'disease symptoms and treatment', 'diagnosis and treatment process', specific 'emotional impact', and 'treatment success factors'. Next, even though the VFQ-25 distinguishes 11 vision-related subscales, this instrument also fails to address the themes 'diagnosis and treatment process', 'emotional consequences' and some of the 'treatment success factors' found to be of significance for chronic uveitis by adult patients. Our findings therefore reveal that - in addition to clinical and 
quality of life outcomes - process factors are also relevant when measuring the impact of this complex and variable condition from a patient perspective.

Next to our main results, there are several findings worth further consideration. First, we note that access to an uveitis specialist familiar with the patient appears highly valued by patients. A trained coordinator may be beneficial to this purpose. Such a person may have added value in improving accessibility, the interdisciplinary monitoring of disease-activities, ensuring timely and accurate referral and the management of in-betweenvisits questions that do not require a visit to the clinic. A second finding worth highlighting is the uncertainty patients experience about short- and long-term disease outcomes. Providing information and clear communication on these matters may help patients to better prepare for the sometimes capricious disease course of chronic uveitis. A third finding for further consideration relates to the difficulties patients experience in coping with prolonged medication. Our findings suggest that better alignment with patients about risks and benefits of specific types and dosages of medication may provide patients with more control and understanding of their treatment. That may have a positive effect on how patients evaluate the outcome of their treatment, as shared decision making about medication can increase patients' satisfaction [23]. This being said, we note that shared decision making in case of chronic uveitis can be complicated by the limited number of prospective randomized controlled trials studying the various systemic medication treatments and the complexity of the disease.

A major strength of this study was the diversity of patients who were selected by stratified sampling from patients' records. The methods used ensured that a wide variety of chronic uveitis patients were included in the focus groups. However, we also note that by deliberately making heterogeneous groups, comparing results between subgroups becomes complex. A limitation of this study is therefore that we can only report about the heterogeneous group of chronic uveitis patients as a whole and not about subgroups e.g., patients diagnosed with ocular sarcoidosis or Birdshot retinochoroidopathy.

In conclusion, we have proposed a conceptual model containing five themes that are important when evaluating the impact of chronic uveitis in adult patients. These themes with their underlying codes can be used to develop a disease specific measurement instrument for adult chronic uveitis patients. With such an instrument patients' disease experiences can be monitored and used to further improve the care provided and their quality of life.

\section{Supplementary information}

Supplementary information accompanies this paper at https://doi.org/10. 1186/s12886-020-01388-y.
Additional file 1. Topic list focus group.

Additional file 2. Final structured analysis matrix.

\section{Abbreviations}

NEI-VFQ-25: 25-item National Eye Institute Visual Function Questionnaire; SF36: SF-36 Health Survey

\section{Acknowledgements}

Not applicable.

\section{Authors' contributions}

$H K, L K, K N$, and AS designed the study. HK and LK carried out the focus groups with support from TM. HK, LK, and AS analysed and interpreted the data. TM analyzed and interpreted the data from a medical practice viewpoint and provided input for clinical implications. LK and AS wrote the manuscript with input from all authors. JB and JK critically revised the manuscript for intellectual content. All authors read and approved the final manuscript.

\section{Funding}

This work was supported by the Netherlands Organisation for Health Research and Development, Topzorg under Grant number 842005001.

\section{Availability of data and materials}

The data that support the findings of this study are available on request from the corresponding author AS. The data are not publicly available due to containing information that could compromise research participant privacy.

\section{Ethics approval and consent to participate}

This study has been approved by the medical ethics committee METC of Erasmus Medical Center (MEC-2017-557). All participants signed informed consent.

\section{Consent for publication}

Not Applicable.

\section{Competing interests}

The authors declare that they have no competing interests.

\section{Author details}

${ }^{1}$ Rotterdam Ophthalmic Institute, Schiedamse Vest 160, Rotterdam 3011 BH, The Netherlands. ${ }^{2}$ Erasmus School of Health Policy \& Management, Erasmus University Rotterdam, Rotterdam, The Netherlands. ${ }^{3}$ Department of

Psychiatry, Section Medical Psychology and Psychotherapy, Erasmus Medical Center, Rotterdam, The Netherlands. ${ }^{4}$ The Rotterdam Eye Hospital, Rotterdam, The Netherlands. ${ }^{5}$ Prince Mohammad Bin Salman School of Business and Entrepreneurship, King Abdullah Economic City, Saudi Arabia.

Received: 12 November 2019 Accepted: 12 March 2020

Published online: 30 March 2020

\section{References}

1. De Smet MD, Taylor SR, Bodaghi B, et al. Understanding uveitis: the impact of research on visual outcomes. Prog Retin Eye Res. 2011;30:452-70. 21 807112. https://doi.org/10.1016/.preteyeres.2011.06.005.

2. Schiffman RM, Jacobsen G, Whitcup SM. Visual functioning and general health status in patients with uveitis. Arch Ophthalmol. 2001;119(6):841-9 PMID: 11405835.

3. Denniston AK, Holland GN, Kidess A, Nussenblatt RB, Okada AA, Rosenbaum JT, Dick AD. Heterogeneity of primary outcome measures used in clinical trials of treatments for intermediate, posterior, and panuveitis. Orphanet J Rare Dis. 2015;10:97. https://doi.org/10.1186/s13023-015-0318-6 PMID: 26286265 .

4. Hui MM, Wakefield D, Patel I, Cvejic E, McCluskey PJ, Chang JH. Visual functioning and health-related quality of life are compromised in patients with uveitis. Ocul Immunol Inflamm. 2017;25(4):486-91. https://doi.org/10. 3109/09273948.2016.1139734 PMID: 27002552. 
5. Denniston AK, Kyte D, Calvert M, Burr JM. An introduction to patientreported outcome measures in ophthalmic research. Eye (Lond). 2014;28: 637-45. https://doi.org/10.1038/eye.2014.41 PMID: 24625379.

6. Mangione CM, Lee PP, Gutierrez PR, Spritzer K, Berry S, Hays RD, National Eye Institute Visual Function Questionnaire Field Test Investigators. Development of the 25-item National Eye institute Visual Function Questionnaire. Arch Ophthalmol. 2001;119(7):1050-8 PMID: 11448327.

7. Dean S, Mathers JM, Calvert M, Kyte DG, Conroy D, Flokard A, Southworth S, Murray Pl, Denniston A. "The patient is speaking": discovering the patient voice in ophthalmology. Br J Ophthalmol. 2017;101:700-8. https://doi.org/ 10.1136/bjophthalmol-2016-309955 PMID: 28455280.

8. Ware JE, Kosinski M, Keller SD. SF-36 physical and mental health summary scales: a user's manual. Boston: Health Assessment Lab; 1994.

9. Suger EA, Venugopal V, Thorne JE, et al. Longitudinal vision-related quality of life for patients with non-infectious uveitis treated with fluocinolone acetonide implant or systematic corticosteroid therapy. Ophthalmology. 2017;124(11):1662-9. https://doi.org/10.1016/j.ophtha.2017.05.015 PMID: 28624167.

10. Braithwaite T, Calvert M, Gray M, Pesudovs K, Denniston AK. The use of patient-reported outcome research in modern ophthalmology: impact on clinical trials and routine clinical practice. Patient Relat Outcome Meas. 2019; 10:9-24. https://doi.org/10.2147/PROM.S162802 PMID: 30774489.

11. Angeles-Han ST. Quality of life metrics in pediatric uveitis. Int Opthalmol Clin. 2015;55(2):93-101. https://doi.org/10.1097/IO.0000000000000067 PMID: 25730622.

12. Lehoux P, Blake D, Daudelin G. Focus group research and "the patient's view". Soc Sci Med. 2006;63:2091-104. https://doi.org/10.1016/j.socscimed. 2006.05.016 PMID: 16797811.

13. Patrick DL, Burke LB, Gwaltney CJ, et al. Content validity--establishing and reporting the evidence in newly developed patient-reported outcomes (PRO) instruments for medical product evaluation: ISPOR PRO good research practices task force report: part 1--eliciting concepts for a new PRO instrument. Value Health. 2011;14(8):967-77. https://doi.org/10.1016/j.jval. 2011.06.014 PMID: 22152165

14. U.S. Department of Health, Human Services Food and Drug Administration, Center for Drug Evaluation and Research, Center for Biologics Evaluation and Research, \& Center for Devices and Radiological Health. Guidance for industry patient-reported outcome measures: use in medical product development to support labelling claims. Rockville: Food and Drug Administration; 2009.

15. Jabs DA, Nussenblatt RB, Rosenbaum JT, et al. Standardization of uveitis nomenclature for reporting clinical data. First international workshop. Am J Ophthalmol. 2005;140:509-16.

16. Carlsen B, Glenton C. What about N? A methodological study of sample-size reporting in focus group studies. BMC Med Res Methodol. 2011;11(1):26. https://doi.org/10.1186/1471-2288-11-26 PMID: 21396104

17. ATLAS.ti. Version 8. Computer software. Berlin: Scientific; 2018.

18. Bowling A. Research methods in health: investigating health and health services. 2nd ed. Berkshire and New York: Open University Press; 2006.

19. Eriksson E, Kovalainen A. Qualitative methods in business research. London: Sage Publications; 2013.

20. Gale NK, Heath G, Cameron E, Rashid S, Redwood S. Using the framework method for the analysis of qualitative data in multi-disciplinary health research. BMC Med Res Methodol. 2013;13:117. https://doi.org/10.1186/ 1471-2288-13-117 PMID: 24047204.

21. Braun V, Clarke V. Using thematic analysis in psychology. Qual Res Psychol. 2006;3(2):77-101. https://doi.org/10.1191/1478088706qp063oa.

22. Green J, Thorogood N. Qualitative methods for health research. 4th ed. London: Sage Publications; 2014. p. 227-9.

23. Dick AD, Rosenbaum JT, Al-Dhibi HA, et al. Guidance on noncorticosteroid systemic Immunomodulatory therapy in noninfectious uveitis. Ophthalmology. 2018;124:757-73. https://doi.org/10.1016/j.ophtha.2017.11. 017 PMID: 29310963

\section{Publisher's Note}

Springer Nature remains neutral with regard to jurisdictional claims in published maps and institutional affiliations.

\section{Ready to submit your research? Choose BMC and benefit from:}

- fast, convenient online submission

- thorough peer review by experienced researchers in your field

- rapid publication on acceptance

- support for research data, including large and complex data types

- gold Open Access which fosters wider collaboration and increased citations

- maximum visibility for your research: over $100 \mathrm{M}$ website views per year

At BMC, research is always in progress.

Learn more biomedcentral.com/submissions 